\title{
Electroclinical Features of Lennox-Gastaut Syndrome in Adulthood and Adolescence
}

\author{
Adriana Ma Goicoechea Astencio, René Andrade Machado ${ }^{*}$, Yudith Merayo², Andrés Rodrigo Solarte Mila ${ }^{3}$, Martha Jiménez Jaramillo ${ }^{4}$ \\ and Juan Felipe Alvarez Restrepo ${ }^{4}$
}

${ }^{1}$ National Institute of Neurology and Neurosurgery, Epilepsy Section, Cuba

${ }^{2}$ Hospital Clínico Quirúrgico de Morón, Ciego de Ávila, Cuba

${ }^{3}$ León XIII Clinic, Antioquia University, Colombia

${ }^{4}$ Neurologic Institute of Antioquia, Colombia

\section{Summary}

Introduction: Lennox- Gastaut Syndrome (LGS) is characterized by seizures which may have inconspicuous semiological features so they may be unrecognized while patients are continuously deteriorating. To confirm its clinical and electrographic characteristics is mandatory, which has therapeutic and prognostic implications. Those features have not been completely elucidated in LGS in adulthood.

Purpose: We performed a descriptive study to investigate seizure types, interictal and ictal EEG characteristics and cognitive outcome in adult LGS subjects.

Methods: We evaluated 28 cases with development impairment and several refractory seizure types, which included tonic seizures, in order to make a screening of LGS. We confirm LGS diagnosis in 24 patients older than 12 years who were assessed by video-EEG, particularly to record seizure types and EEG findings as well as cognitive outcome.

Results: During this stage of the disease, all patients presented tonic seizures (TS) during wakefulness and sleep, 12/24 had atypical absences, more rarely other seizure types. Some cases (5/24) have evolved from West syndrome and in $62.5 \%$ aetiology was cryptogenic. Interictal EEG showed normal background activity in 13/24 patients, slow spike-wave discharges during wakefulness was diffuse just in 4/24; bursts of diffuse fast rhythms (DFR) in sleep were seen in all patients. A moderate to severe cognitive impairment was observed in 18/24 patients, but all of them experience deterioration after the epilepsy had begun.

Conclusions: In adult LGS patients a standard waking EEG may be normal. TS during sleep and the presence of DFR are paramount to confirm the diagnosis.

Keywords: Lennox-Gastaut syndrome; Electro-clinical features; Fast rhythms; Adult Lennox Gastaut syndrome; Cognitive

\section{Introduction}

Lennox-Gastaut Syndrome (LGS) is a rare childhood epileptic encephalopathy estimated to affect up to 2 in 10,000 people in Europe [1]. The prevalence of LGS among children with epilepsy has been reported to be 3-6\% [2-4], although this estimate probably reflects a very broad definition of the syndrome. If strict diagnostic criteria are used, the prevalence is $3 \%$ [5]. Males are affected up to five times more often than females [1]. Symptoms usually start between 3 and 5 years of age but may appear earlier, while onset after 10 years of age is rare [6]. Although there have been reports of LGS being diagnosed in adults, the appropriateness of such diagnoses may be questioned [7-9].

This syndrome has been clearly defined, in children, by an electroclinical triad: many types of seizures refractory to antiepileptic treatment, which include tonic, atypical absences and atonic, with tonic seizures during sleep as a constant manifestation; mental and behavioural impairment and an electrographic pattern characterized by generalized slow spike-wave in wakefulness and diffuse fast rhythms, with $10-20 \mathrm{~Hz}$ frequency, in sleep $[8,10]$.

LGS was adopted by the ILAE Commission on Classification and Terminology in 2001, as an Epileptic Encephalopathy, condition in which the epileptiform abnormalities themselves are believed to contribute to progressive disturbance of cerebral function [11]. Among the epilepsies included in this group, this is one of the most frequent in childhood, with well defined clinical and electrical features at this moment and which usually evolves into another type of focal or multifocal epilepsy, reason for which most of those patients -in adolescence and adulthood- receive antiepileptic treatment for focal epilepsies, contraindicated in LGS if the syndrome remains. Besides, there are other focal symptomatic epilepsies with axial tonic seizures, whose epileptogenic area are localized in supplementary sensoriomotor cortex and severe epilepsy with multiple independent spikes foci, very difficult to distinguish of this syndrome in clinical grounds $[12,13]$. Furthermore, patients with diffuse fast rhythms in sleep without tonic seizures ore even other seizure types and without cognitive deterioration that suggest LGS diagnosis [14].

Orell-Daurella in 1967, described a form of development of LGS in older ages which he denominated at that moment, late-onset slow spike-wave. His patients did not presented the same characteristics of those who had early-onset slow spike-wave, because astatic seizures were not so frequent, non-convulsive status epilepticus was not as usual

*Corresponding author: René Andrade Machado, National Institute of Neurology and Neurosurgery, Epilepsy Section, Cuba, E-mail: renemachado@infomed.sld.cu

Received April 16, 2013; Accepted June 17, 2013; Published June 25, 2013

Citation: Astencio AMG, Machado RA, Merayo Y, Mila ARS, Jaramillo MJ, et al. (2013) Electroclinical Features of Lennox-Gastaut Syndrome in Adulthood and Adolescence. J Neurol Neurophysiol S2: 008 doi:10.4172/2155-9562.S2-008

Copyright: @ 2013 Astencio AMG, et al. This is an open-access article distributed under the terms of the Creative Commons Attribution License, which permits unrestricted use, distribution, and reproduction in any medium, provided the original author and source are credited. 
and the diffuse slow spike-wave electrical pattern tended to disappear. Although the electroclinical features are very well defined in children, the assessment of longitudinal studies in adults has been very poor. That's why there is not consensus about SLG criteria in adulthood. One of the most relevant works at this respect is the already mentioned Orell-Daurella's report, of 22 cases with late-onset slow spike-wave [15]. Ferlazzo et al. has also developed a multicentric research about electroclinical characteristics of 27 adult patients with this syndrome, who were studied from 1960 to 1976 [16]. This same author reported LGS in some older cases with Down syndrome [17] and more recently, has been published a Spanish study of 12 cases about the evolution of LGS in adult ages [18].

On the other hand, it has been proposed as an electroclinical syndrome that makes detection of clinical and electrographic characteristics of the syndrome itself, mandatory to establish a certain diagnosis [19]. That's why is so remarkable to accurately know the electroclinical features that characterized LGS when it starts or continues in adolescence or adulthood, which has prognostic and therapeutic consequences.

In Cuba, any research about LGS has been carried out. Having a health care system that guarantee the survival of patients with encephalopathy until older ages, is probably that we have a population of persons older than 12 years, in whom LGS has begun or persisted, which has motivated us to study electroclinical characteristics of this syndrome in adolescents and adults.

\section{Purpose} adults.

To describe the electroclinical features of LGS in adolescents and

\section{Patients and Methods}

The study was developed, from May 2010 to January 2011. A screening of LGS was carried out among those patients who were evaluated, in the National Institute of Neurology and Neurosurgery of Cuba and Antioquia University in Colombia, for drug-resistant epilepsy. We initially screened all patients with refractory epilepsy presenting tonic seizures and we suspected LGS diagnosis when patients had several seizure types and developmental deterioration after epilepsy onset.

Definitive diagnosis of LGS was based on the following criteria: multiple generalized seizure types including tonic seizures; diffuse fast rhythms and/or recruiting rhythms on the EEG on non-REM sleep and mental deterioration associated with the onset of epilepsy. We did not include patients with interictal electrographic activity characterized by multifocal spikes, focal delta activity different to delta with maximum in the frontal region that typically differentiates LGS from severe epilepsy with multiple independent spikes foci. Age older than 12 years at recruiting point was also an inclusion criterion.

Patients were assessed in video-EEG, during wakefulness and sleep states. Information about seizure types were obtained from epilepsy diaries, relatives' descriptions and video-EEG analysis. Additional clinical data collected including gender, personal antecedents, current age and age at seizure onset, diagnosis before the evaluation, and existence of neurodevelopment retardation previous to the beginning of seizures were obtained from clinical examination to patients and interviews to patients and relatives. Current severity of mental retardation was assessed by means of Wechsler Adult Intelligence Scale (WAIS) and Wechsler Intelligence Scale for Children, Revised (WISC-R), for those who were younger than 16 years.
All patients had undergone EEG examination during wakefulness and physiologic sleep, including at least surface EMG of deltoid muscles, with the now known as the International 10-20 system, with additional zygomatic electrodes and reference located according to above mentioned montage.

Two patients with clinical suspicion of LGS were excluded because their behaviour didn't allow video-electrographic assessment.

\section{Results}

\section{Screening phase}

Twenty-four out of 28 patients evaluated, in whom LGS was suspected, fit the inclusion criteria. One of the rests had a biopercular syndrome and the other three had focal frontal epilepsy.

\section{Age at onset}

With respect to distribution of patients according to age at onset, in the majority of them Epilepsy started between 1 and 5 years (66.7\%). Just 2 patients debuted after 10 years of age (Table 1).

Age at recruiting point: At the point of enrolment most of the patients (62.4\%) were aged between 16 and 30 years (Table 2).

Sex: The proportion $\mathrm{F} / \mathrm{M}=1$ (12 females and 12 males).

Aetiology: With respect to aetiology, 13/24 patients were cryptogenic and 9/24, were symptomatic: 1 had tuberous sclerosis, 1 had a mitochondrial encephalomyopathy, 1 had a previous history of meningoencephalitis, 5 had antecedent of perinatal asphyxia and 1 of them had a parietal cortical dysplasia (Table 3 ).

Neurodevelopment before epilepsy onset: Sixteen out of 24 patients had a normal neurodevelopment before the onset of epilepsy, who belonged to cryptogenic group. However, all of them experienced

\begin{tabular}{|c|c|}
\hline Age at Epilepsy onset (years) & $\# \mathbf{( \% )}$ \\
\hline $0-5$ & $16(66.7)$ \\
\hline $6-10$ & $6(25.0)$ \\
\hline $11-15$ & $1(4.2)$ \\
\hline $16-20$ & $1(4.2)$ \\
\hline Total & $\mathbf{2 4 ( 1 0 0 )}$ \\
\hline
\end{tabular}

Table 1: LGS patients according to age at epilepsy onset.

\begin{tabular}{|c|c|}
\hline Age at recruiting point (years) & $\#(\%)$ \\
\hline $12-15$ & $3(12.5)$ \\
\hline $16-20$ & $5(20.8)$ \\
\hline $21-25$ & $5(20.8)$ \\
\hline $26-30$ & $5(20.8)$ \\
\hline $31-35$ & $1(4.2)$ \\
\hline $36-40$ & $2(8.3)$ \\
\hline+40 & $3(12.5)$ \\
\hline Total & $\mathbf{2 4 ( 1 0 0 )}$ \\
\hline
\end{tabular}

Table 2: LGS patients according to age at evaluation.

\begin{tabular}{|c|c|}
\hline Etiology of symptomatic cases & $\mathbf{N}(\%)$ \\
\hline Perinatal asphyxia & $5(55.6)$ \\
\hline Tuberous sclerosis & $1(11.1)$ \\
\hline Mitochondrial encephalomyopathy & $1(11.1)$ \\
\hline Meningoencephalitis & $1(11.1)$ \\
\hline Parietal cortical dysplasia & $1(11.1)$ \\
\hline Total & $\mathbf{9 ( 1 0 0 )}$ \\
\hline
\end{tabular}

Table 3: Principals etiologies found in our serie in symptomatic cases. 
deterioration of cognition and/or behaviour after the beginning of epilepsy. According to the IQ at the evaluation 10/24 and $8 / 24$ had a severe and a moderate mental retardation, respectively, while $4 / 24$ had a minor mental impairment. There were even two cases with a borderline IQ (Table 4).

West syndrome past history: There were 5 out of 24 patients who evolved from a West Syndrome, who corresponded to the group of symptomatic aetiology.

Seizure types: Atypical absences persisted in 12/24 patients, astatic in $3 / 24$, generalized tonic-clonic in $4 / 24$, myoclonic in $7 / 24$, atonic in $4 / 24$ and focal seizures in 11/24. All cases had axial tonic seizures, which was an inclusion criterion (Figure 1). During video-EEG assessment one patient had atypical absences status which corresponded to that with diagnosis of mitochondrial encephalomyopathy. There was also another one with tonic status whose mother reported to be controlled before enrolment.

Interictal electrographic pattern: Most of our patients (54.2\%) showed a normal background activity in the interictal EEG during wakefulness. In only $4(16.7 \%)$ of them the diffuse slow spike-wave pattern, typical of childhood, was retained (Figure 2).

In all our patients the presence of diffuse fast rhythms in non-REM sleep was confirmed and, as all of them had also tonic seizures during sleep, $100 \%$ of cases also showed recruiting rhythms (Figure 3).

Previous diagnosis: Just 4/24 patients had a correct diagnosis of LGS before the study (Table 5).

Antiepileptic drugs used at evaluation point: As most of the patients had an incorrect diagnosis before the study, they had also inadequate treatments for LGS, which may be even contraindicated. Most of them were also under treatment with neuroleptics because of

\begin{tabular}{|c|c|}
\hline Mental impairment (IQ) & $\mathbf{N}(\%)$ \\
\hline Severe $(I Q<30)$ & $10(41.7)$ \\
\hline Moderate $(30<\mid \mathrm{Q}<50)$ & $8(33.3)$ \\
\hline Slight $(50<\mid \mathrm{Q}<70)$ & $4(16.7)$ \\
Borderline $(\mathrm{IQ} \sim 70)$ & $2(8.3)$ \\
\hline Total & $\mathbf{2 4}(\mathbf{1 0 0})$ \\
\hline
\end{tabular}

Table 4: LGS patients according to Intelligence Quotient. behaviour impairment, although there were 7 who did not need this kind of medication (Table 6).

\section{Discussion}

\section{Screening phase (Differential diagnosis)}

Twenty-four out of 28 patients evaluated, in whom LGS was suspected, fit the inclusion criteria. One of the rests had reflex and spontaneous tonic seizures and astatic seizures due to a biopercular syndrome. The other three had astatic seizures, asymmetric tonic seizures and episodes characterized by impairment of consciousness which corresponded to complex partial seizures and interpreted as atypical absences. Focal epilepsy with an epileptogenic area estimated in mesial frontal lobe (supplementary cortex) was diagnosed in this group of patients. Lipinski (1977) reported some cases with late onset astatic seizures in whom LGS diagnosis was established, but tonic seizures or burst of generalized rapid EEG rhythms during sleep were missing [9]. This confirms how difficult could be the differentiation between those syndromes in clinical grounds.

\section{Age at epilepsy onset and at evaluation}

At epilepsy onset most of the patients were younger than 5 years old while only 2 of them were older than 10 . This corresponds with literature reports. Usually LGS debuts between 2 and 8 years, with very few cases in whom this epileptic syndrome starts after 10 years of age [6]. There are few reports of LGS patients beginning after this age $[7,8]$ and the investigations developed in adult LGS subjects did not evaluate this point, making comparisons among studies impossible. There is a research developed by about LGS in patients with Down Syndrome, where in most of them (62\%) epilepsy started after 8 years of age [17]. Authors attributed this finding to a possible delay of frontal lobe maturation, or early onset of degenerative changes in the same areas, because, blood flow changes during brain development contributes to the age-dependency of some syndromes such as West syndrome, which seems to be linked to maturation of the posterior areas, and LGS, which appears to be related to maturation of the anterior brain. Alas, it might explain the late age at onset of LGS in trisomy 21 with respect to what is usually seen with most etiologies [17]. Lipinski also reported LGS patients with a late epilepsy onset, but those cases did not show the typical features that define this syndrome, so the certainty of this diagnosis could be debated [9].

\section{Seizure types}

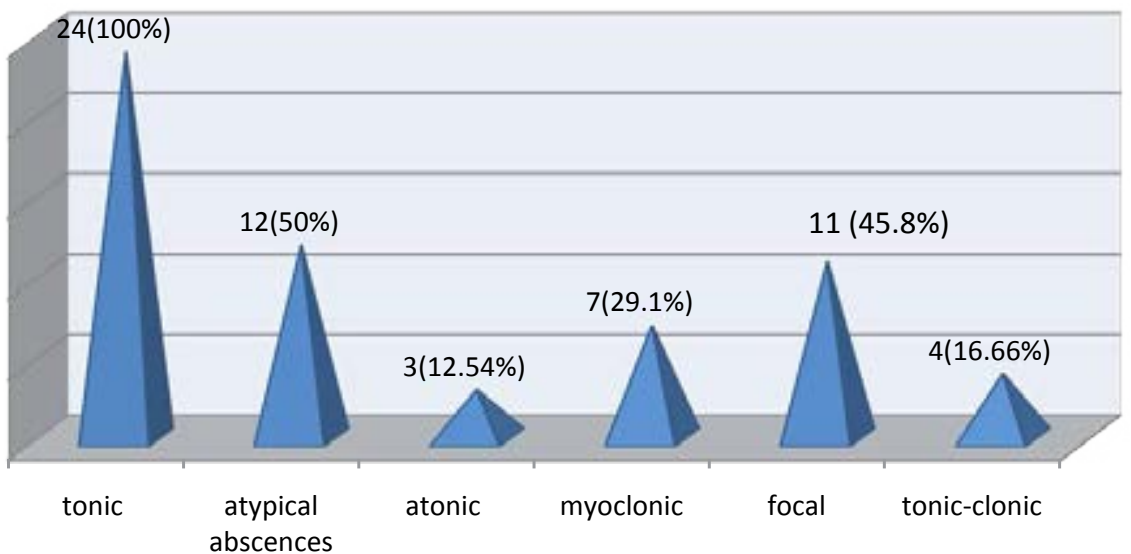

Figure 1: LGS patients according seizure types. 


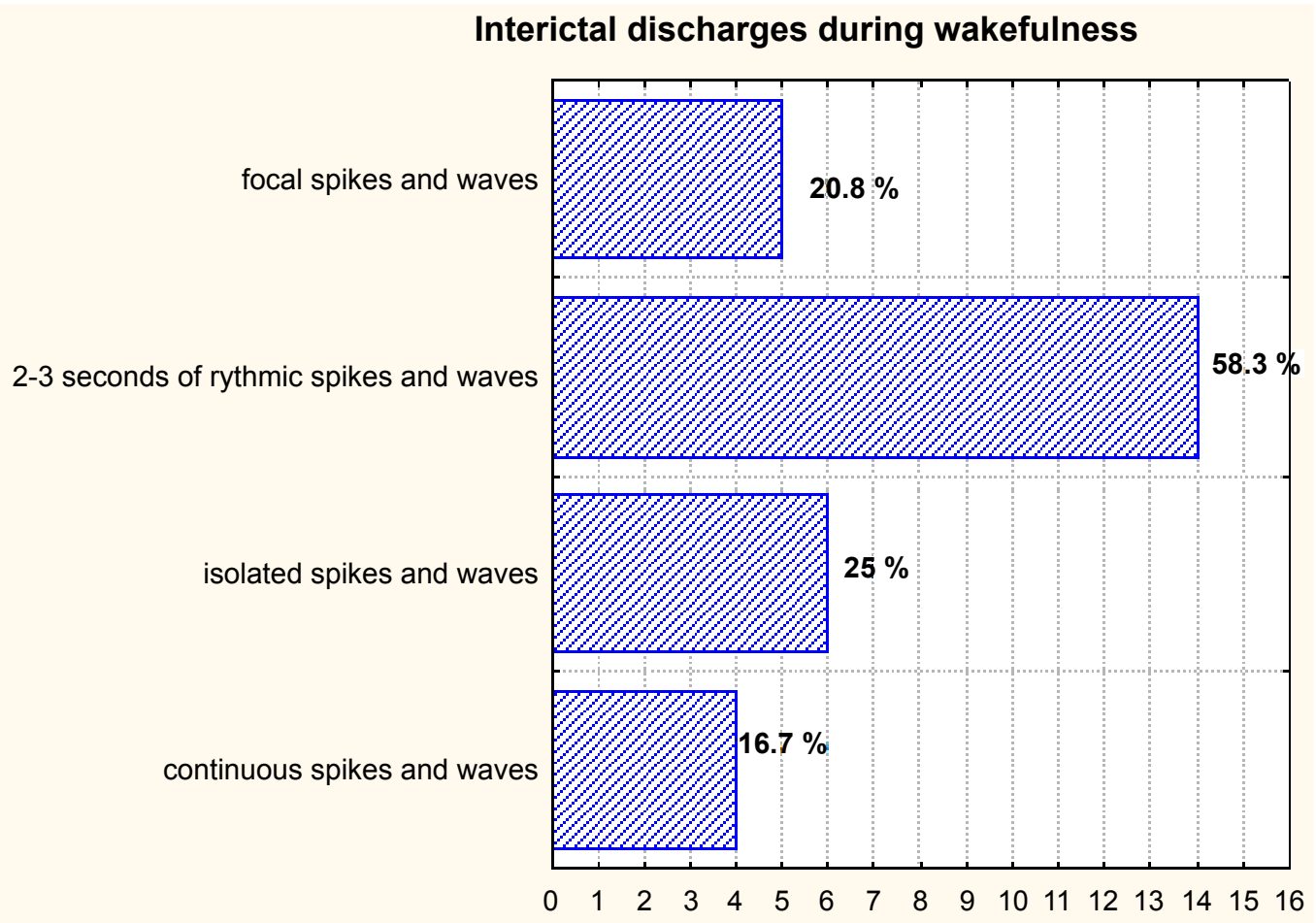

$\dddot{Z}$ percent of interictal discharges

Figure 2: LGS patients according spike-wave interictal pattern during wakefulness.

At recruiting time the majority of cases were aged between 16 and 30 years, although there were 3 patients (12.5\%) older than 40 years. This finding shows that patients with LGS could survive for many decades and that the syndrome itself could be retained.

\section{Sex}

The proportion $\mathrm{F} / \mathrm{M}=1$ (12 females and 12 males). This result disagrees with literature, with predominance of males in the studies developed in children and also in adults, which could be due to selection bias. Nevertheless, there is a report in which female gender was even more frequent [16].

\section{Aetiology}

With respect to aetiology, $13 / 24$ were cryptogenic (62.5\%) and 9/24 (37.5\%), were symptomatic: 1 had tuberous sclerosis, 1 had a mitochondrial encephalomiopathy, 1 had a previous history of meningoencephalitis, 5 had antecedent of perinatal asphyxia and 1 of them had a parietal cortical dysplasia. This coincides with findings of investigations carried out in adults, where cases without a demonstrated aetiology were more frequently found $[15,16]$, without differences of prognosis or evolution in these groups of patients.

Several studies showed no significant differences between cryptogenic and symptomatic cases in terms of evolution of seizure types or frequency, EEG findings, or response to treatment. Symptomatic LGS patients tend to have more seizure types, but cryptogenic aetiology did not decrease the risk of poor [20]. Ferlazzo's results are also in accordance with those findings [16].

\section{Neurodevelopment impairment}

Sixteen out of 24 patients had a normal neurodevelopment before the onset of epilepsy, who belonged to cryptogenic group. However, all of them experienced deterioration of cognition and/or behaviour after the beginning of epileptic seizures, although two of those patients (8.3\%) had an IQ>70 (borderline).

It's been reported that in adult LGS patients, up to $20 \%$ of them may have a normal IQ [10] and Goldsmith et al. (2000) found that 8\% LGS subjects did not display mental retardation [20]. However, the prognosis in cognition is usually poor and literature data report up to $43 \%$ of cognitive impairment in cryptogenic patients and $76 \%$ in symptomatic ones [21]. Yagi found that 12 out of 102 patients with LGS worked normally and 36 had part-time or sheltered jobs [22]. In Ferlazzo's series, 26/27 patients at the end of follow-up had moderate to severe cognitive impairment and in Rodríguez's study, 11/12 had a severe mental retardation $[16,18]$.

While Ogawa et al. showed that the presence of atypical absences at the onset of the disease and the persistence of frequent epileptiform discharges at follow-up, represented strong predictors of both poor cognitive and seizure outcome [23]. One of our patients with a normal IQ had 37 years evolution of the disease, with an incorrect diagnosis of temporal lobe epilepsy refractory to antiepileptic drugs, under treatment with carbamazepine and benzodiazepines, which are known drugs that get LGS worst. This patient consequently suffered multiple atypical absences daily, and abundant complexes of slow spike-wave were seen in her EEG, alas we consider there should be other predictors of intellectual deterioration. Seizures in this patient started when she was 10 years old, after critical period for neurodevelopment, which may be a possible explanation.

Another retrospective investigation identified 4 independent risk factors for severe mental retardation: non-convulsive status epilepticus, previous diagnosis of West Syndrome, symptomatic aetiology and early 
Citation: Astencio AMG, Machado RA, Merayo Y, Mila ARS, Jaramillo MJ, et al. (2013) Electroclinical Features of Lennox-Gastaut Syndrome in Adulthood and Adolescence. J Neurol Neurophysiol S2: 008 doi:10.4172/2155-9562.S2-008
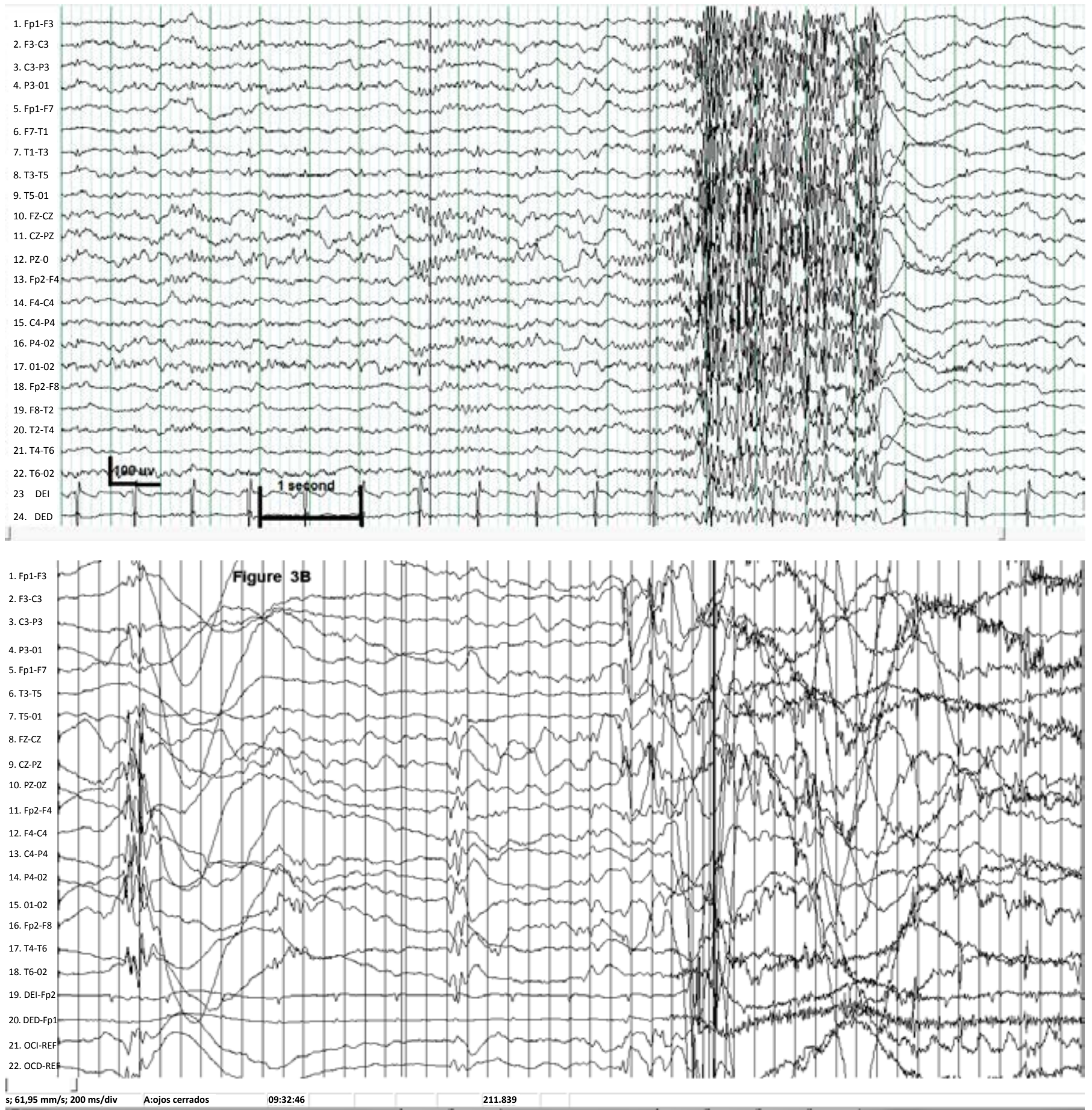

Figure 3: Polygraphic study in one of our patients. Patient FGR 24 years old. Figure 3A shows fast rhythms during stage 2 non-REM sleep. Note the presence of normal sleep spindles. Figure 3B (the same patient) shows a diffuse fast rhythm without deltoid contraction (interictal pattern) followed by a typical asymmetric tonic seizure. See right deltoid tonic contraction before mild left deltoid contraction. DE means deltoid muscle: D (right) I (left). OC refers to oculograms: $\mathrm{D}$ (right), $\mathrm{L}$ (left). Figure $3 \mathrm{C}$ see the normal background in one of our patient.

age of onset of seizures. These are probably also the most important prognostic criteria for seizure control and social outcome [24].

\section{West syndrome past history}

There were 5 (20.8\%) out of 24 patients who evolve from a West Syndrome, who belonged to the group of symptomatic aetiology.
Data collected in literature report that approximately $20 \%$ may have a preceding history of West syndrome. In Rodríguez's series it occurred in $3 / 12$ cases $(25 \%)$ [18]. Nevertheless, it is remarkable that although in trisomy 21 infants, West syndrome is the epilepsy most frequently found, in any patient reported by Ferlazzo with late-onset LGS in Down syndrome, had this antecedent [17]. 


\begin{tabular}{|c|c|}
\hline Previous diagnosis & N (\%) \\
\hline Focal epilepsy & $17(70.8)$ \\
\hline Lennox-Gastaut syndrome (LGS) & $4(16.7)$ \\
\hline Generalized epilepsy other than LGS & $3(12.5)$ \\
\hline Total & $\mathbf{2 4}(\mathbf{1 0 0})$ \\
\hline
\end{tabular}

Table 5: LGS patients according to diagnosis before Video-EEG evaluation.

\begin{tabular}{|c|c|c|}
\hline AEDs & N (\%) & Mean dose \pm SD (mg/daily) \\
\hline Valproic acid+clonazepam & $12(50)$ & $1890 \pm 123.5+3 \pm 0.55$ \\
\hline Carbamazepine+Lamotrigine & $6(25)$ & $450 \pm 43.2+300 \pm 43.5$ \\
\hline Carbazepine+clonazepam & $2(8.3)$ & $550 \pm 75.3+4 \pm 0.2$ \\
\hline Valproic acid+carbamazepine & $4(16.6)$ & $1890 \pm 234.7+450 \pm 54.2$ \\
\hline \multicolumn{2}{|c|}{ NEUROLEPTICS } \\
\hline Quetiapine (200 mg) & $5(20.8)$ & $150 \pm 25.7$ \\
\hline Olanzapine (10 mg) & $7(29.2)$ & $15 \pm 2.1$ \\
\hline Risperdal (2 mg) & $5(20.8)$ & $5 \pm 0.76$ \\
\hline Without neuroleptics & $7(29.2)$ & 0 \\
\hline
\end{tabular}

Table 6: Antiepileptic and neuroleptic medications in LGS patients at evaluation.

\section{Seizure types at recruiting point}

Atypical absences persisted in $12 / 24$ patients, astatic in $3 / 24$, generalized tonic-clonic in 4/24, myoclonic in $7 / 24$, atonic in $4 / 24$ and focal seizures in 11/24. All cases had axial tonic seizures (an inclusion criterion) at least during sleep; even in those who reported to be seizure free at evaluation, this seizure type was observed at this state.

In relation to seizure type, Rodríguez et al. observed that $100 \%$ of cases suffered tonic seizures and atypical absences; drop-attacks were also very common, what was not found in our study. Nine (75\%) presented generalized tonic-clonic seizures; $5(41.7 \%)$, while myoclonic and focal seizures were seen just in one patient [18]. Ferlazzo et al. (2010) found a similar frequency of seizures as our research [16]. All their patients had tonic seizures during sleep, with atypical absences as the second most frequent seizure type, but with a great reduction of them in time [16]. Ferlazzo et al. in their series of late-onset LGS associated with trisomy 21, seizure type's frequency was also comparable [17]. Yagi also found that tonic seizures, especially during sleep, were the most resistant seizure type and persisted in nearly all patients; in addition, $20 \%$ of his patients also had atypical absences or astatic seizures and only $8 \%$ were seizure-free [22]. This information should be analyzed cautiously, because as was already mentioned, all the patients whose relatives reported to be seizure free actually were still suffering for underappreciated tonic seizures in sleep.

Since daytime tonic seizures, astatic ones and atypical absences may disappear over the years, adult LGS subjects may not retain the same tendency to fall as compared to childhood or youth, thus making heavy or specific (rufinamide, callosotomy, etc.) treatments to prevent drop attacks, questionable or unnecessary in some patients [16].

In our patients, tonic seizures were characterized by an axial contraction, with shoulders elevation and flexion of the head, sometimes accompanied by flexion of inferior extremities, of very brief duration; atypical absences could be preceding or following them. In some cases, video-EEG analysis only showed an awakening, an ocular retroversion or just a mimic automatism, with the correspondent recruiting rhythm on the EEG; so these seizures may be underappreciated.

Two patients had also reflex tonic seizures to auditory stimuli and spontaneous (more frequent) ones. According to some authors, reflex seizures are rarely found in patients with LGS, and they are usually related to brain lesions, but those cases were cryptogenic [17].
Kohsaka et al. disclosed a widespread (both ventral and dorsal) and synchronized activation of the brainstem preceding the emergence of fast rhythms. Both parameters (amplitude and area) of both wave -III and V- of auditory evoked potentials gradually increased, reaching their maxima before the onset of diffuse fast rhythms in the scalp EEG [25]. Those results suggest that sounds could activate the brainstem and precipitate axial tonic seizures, as was the case of these patients.

It is notorious that $9 / 13(70 \%)$ patients with trisomy 21 and LGS had also reflex seizures [17]. Such attacks were triggered by sudden unexpected sensory stimulations (mainly noise and touch) in six patients, contact of water with the face (one patient), contact of the glass with the mouth (one patient), emotions or getting up the stairs of staircases (one patient). Reflex seizure types were tonic (eight patients), atypical abscences (one patient), myoclonic (one patient), GTCS (one patient), drop attacks (one patient). Indeed, in trisomy 21 patients, neurophysiologic studies have shown increased amplitude of sensory evoked potentials that were attributed to a central deficit of inhibition of different stimuli, which would lead to an excessive proprioceptive outflow to a hyperexcitable motor cortex, precipitating a seizure [17] We did not performed sensory evoked potentials to our patients, alas the likelihood for this to be another explanation for their reflex tonic seizures could not be discarded. Otherwise, patients with trisomy 21 exhibit a dysfunction of nucleus reticularis pontis caudalis that causes an exaggerated startle response leading to an increase of proprioceptive feedback to a hyperexcitable motor cortex, precipitating a seizure. It reveals an impairment of pontine functioning, which could lead to hyperexcitation at this level, causing sudden noises to originate a tonic seizure as Kohsaka et al. reported [25].

\section{Electrographic pattern}

Most of our patients (54.2\%) showed a normal background activity in the interictal EEG during wakefulness. In only $4(16.7 \%)$ of them the diffuse slow spike-wave pattern, typical of childhood, was retained; 14 (58.3\%) patients had brief bursts of slow spike-wave, 6 (25\%) showed isolated discharges and in $5(20.9 \%)$ of them the spike-wave complexes were not generalized but focal.

When Orell-Daurella described the cases with late-onset slow spike-wave, he reported that they didn't show the diffuse slow spikewave pattern, with a great disturbance of background activity, as it was seen in children. On the contrary, he found that spike-wave complexes appeared in bursts, in isolated form or even focal, with a gradual tendency to the normalization of EEG basic rhythms [15]. In Yagi's LGS series, diffuse slow spike-wave during waking state disappeared first, but multiple spike-and-wave complexes during sleep were seen before the disappearance of all epileptic EEG discharges in patients in whom seizures improved [20]. Hughes and Patil (2002) evaluated the EEG evolution in 64 LGS patients with a follow-up $>16$ years. The disappearance of diffuse spike-wave pattern usually occurred after the age of 16 years while in $95 \%$ of patients background activity gradually slowed and focal discharges remained the only interictal epileptic EEG abnormality. In Ferlazzo's patients, the evolution of EEG findings was characterized by the normalization of background activity in $44 \%$ as well as a clear reduction of epileptiform discharges during wakefulness in $74 \%$ over the years [16]. Those results are in disagreement with Rodríguez's findings, who observed a slow and disturbed activity with frequent intermixed generalized slow spike-wave discharges, as it is seen in childhood [18].

Interictal EEG may be almost normal, with a significant reduction of epileptiform paroxysms during wakefulness. Also during sleep 
interictal background activity may be normal. In fact, 10/24 cases showed normal sleep transients with presence of symmetric vertex sharp waves in stage 1 non-REM sleep and also symmetric and well structured sleep spindles and $\mathrm{K}$ complexes in stage 2 non-REM sleep. Nevertheless, in all our patients the presence of diffuse fast rhythms in non-REM sleep was confirmed and, as all of them had also tonic seizures during sleep, $100 \%$ of cases also showed recruiting rhythms, in accordance with the previous studies [15-18, 22,23].

The presence of recruiting rhythms is a criterion for diagnosis of LGS in children and is retained also in adults. This aspect is crucial when evaluating adult LGS patients. Indeed, a standard EEG may be normal and thus, not significant of the real evolution of patients' seizure disorder or even equivocal, showing focal abnormalities. Hence, only assessing patients during sleep, including EEG and EMG (essential to detect tonic seizures), we can ascertain the correct diagnosis of LGS in these groups of ages [16].

\section{Previous diagnosis}

Just 4/24 patients had a correct diagnosis of LGS before the study, while 3/24 had the diagnosis of Progressive Myoclonic Epilepsy (MERRF and Unverricht-Lundborg disease); a focal symptomatic epilepsy was the diagnosis in most of them (17/24).

Patients had multiple seizure types, with continuous deterioration and some of them had also myoclonic seizures. Three of the cases had, besides cognitive impairment, deterioration of neurological function too. One of them was wheelchair bound because of a mitochondrial encephalopathy with documented defects of I, III and IV complexes of respiratory chain. Two other patients had ataxia due to cerebellar atrophy caused by phenytoin employment. We consider these were the reasons for establishing the diagnosis of progressive myoclonic epilepsy (encephalopathy with epilepsy instead of epileptic encephalopathy) in those patients, many years before the evaluation.

Clinical distinction between atypical absences and complex partial seizures may be very difficult and, in this context, where astatic seizures (extremely frequent in childhood) tend to disappear and axial tonic seizures may be underappreciated, LGS could not be even thought and the diagnosis of a focal epilepsy could be very logical, maxima if the interictal slow spike-wave pattern could be focal and tonic axial seizures may be asymmetric, which may suggest the existence of a mesial frontal lobe epilepsy with epileptogenic estimated area in supplementary sensoriomotor cortex. In fact, 3 of the patients evaluated at the beginning of the screening, with a clinical suspicion of LGS, actually had this epileptic syndrome.

We consider that one of the most interesting points in this research is that we had the possibility of making a correct diagnosis in a significant group of patients, who were receiving inadequate treatments for LGS, which contribute to further deterioration. The other thing we could ascertain is that the criteria for LGS diagnosis at those group of ages is almost the same as in children: multiple seizure types which include tonic seizures, cognitive regression after epilepsy onset, the presence of recruiting rhythms and interictal pattern of slow spike-wave. It is true just in part, because tonic seizures have different semiology, astatic and atypical abscences seizures are less prominent and interictal EEG, although actually shows slow spike-wave discharges, the diffuse pattern that we usually expect is rarely seen. Alas, when the diagnosis of LGS is suspected an EEG during sleep should be performed.

\section{Conclusions}

1. Tonic seizures, although with different semiology, especially during sleep, remain in all patients. So they are a prerequisite for diagnosis of LGS at these ages.

2. Interictal EEG may show a normal background activity, with disappearance of diffuse slow spike-wave pattern, although slow spike-wave discharges tend to persist in some of their forms.

3. Besides recruiting rhythms, diffuse fast rhythms in non-REM sleep are retained in all cases; alas their presence is mandatory for LGS diagnosis in adolescents and adults.

4. As there is a tendency for disappearance of astatic seizures as well as electrographic pattern characteristic of LGS in childhood during wakefulness and tonic seizures may be unrecognized or with asymmetric component, performance of EEG during sleep is absolutely necessary to establish a definitive diagnosis of LGS in adolescence and adulthood.

\section{References}

1. Tidy C Lennox-Gastaut Syndrome. EMIS. Available from: http://www.patient co.uk/showdoc/40001739; 24 Nov 2004 [accessed 5.03.09].

2. Cavazzuti GB (1980) Epidemiology of different types of epilepsy in school age children of Modena, Italy. Epilepsia 21: 57-62.

3. Sidenvall R, Forsgren L, Heijbel J (1996) Prevalence and characteristics of epilepsy in children in northern Sweden. Seizure 5: 139-146.

4. Markand ON (2003) Lennox-Gastaut syndrome (childhood epileptic encephalopathy). J Clin Neurophysiol 20: 426-441.

5. Stephani $U$ (2006) The natural history of myoclonic astatic epilepsy (Doose syndrome) and Lennox-Gastaut syndrome. Epilepsia 47 Suppl 2: 53-55.

6. Bladin PF (1985) Adult Lennox Gastaut syndrome: features and diagnostic problems. Clin Exp Neurol 21: 93-104

7. Bladin PF (1985) Adult Lennox Gastaut syndrome: patients with large foca structural lesions. Clin Exp Neurol 21: 105-114.

8. Wheless JW, Constantinou JE (1997) Lennox-Gastaut syndrome. Pediat Neurol 17: 203-211.

9. Lipinski CG (1977) Epilepsies with astatic seizures of late onset. Epilepsia 18: 13-20.

10. Arzimanoglou A, French J, Blume WT, Cross JH, Ernst JP, et al. (2009) Lennox-Gastaut syndrome: a consensus approach on diagnosis, assessment management, and trial methodology. Lancet Neurol 8: 82-93.

11. Blume WT, Lüders HO, Mizrahi E, Tassinari C, van Emde Boas W, et al. (2001) Glossary of descriptive terminology for ictal semiology: report of the ILAE task force on classification and terminology. Epilepsia 42: 1212-1218.

12. Berg AT, Blackstone NW (2006) Concepts in classification and their relevance to epilepsy. Epilepsy Res 70 Suppl 1: S11-19.

13. Lüders HO, Acharya J, Alexopoulos A, Baumgartner C, Bautista J, et al. (2006) Are epilepsy classifications based on epileptic syndromes and seizure types outdated? Epileptic Disord 8: 81-85

14. Halász P, Janszky J, Barcs G, Szucs A (2004) Generalised paroxysmal fast activity (GPFA) is not always a sign of malignant epileptic encephalopathy. Seizure 13: 270-276.

15. Oller-Daurella L (1967) Síndrome de Lennox y sus diferentes formas clínicas y electroencefalográficas. Revisión basada en un centenar de casos personales de punta-onda lenta difusa. Barcelona: Espaxs.

16. Ferlazzo E, Nikanorova M, Italiano D, Bureau M, Dravet C, et al. (2010) Lennox-Gastaut syndrome in adulthood: clinical and EEG features. Epilepsy Res 89: 271-277.

17. Ferlazzo E, Adjien CK, Guerrini R, Calarese T, Crespel A, et al. (2009) LennoxGastaut syndrome with late-onset and prominent reflex seizures in trisomy 21 patients. Epilepsia 50: 1587-1595

18. Rodriguez-Rodriguez S, Salas-Puig J, Alvarez-Carriles JC, TempranoFernandez T, Anton-Gonzalez C, et al. (2011) [Development of LennoxGastaut syndrome in adulthood]. Rev Neurol 52: 257-263. 
Citation: Astencio AMG, Machado RA, Merayo Y, Mila ARS, Jaramillo MJ, et al. (2013) Electroclinical Features of Lennox-Gastaut Syndrome in Adulthood and Adolescence. J Neurol Neurophysiol S2: 008 doi:10.4172/2155-9562.S2-008

Page 8 of 8

19. Berg AT, Berkovic SF, Brodie MJ, Buchhalter J, Cross JH, et al. (2010) Revised terminology and concepts for organization of seizures and epilepsies: report of the ILAE Commission on Classification and Terminology, 2005-2009. Epilepsia 51: $676-685$.

20. Goldsmith IL, Zupanc ML, Buchhalter JR (2000) Long-term seizure outcome in 74 patients with Lennox-Gastaut syndrome: effects of incorporating MRI head imaging in defining the cryptogenic subgroup. Epilepsia 41: 395-399.

21. Genton P, Dravet C (2008) Lennox-Gastaut syndrome. In: Engel JJr, Pedley TA, eds. Epilepsy: a comprehensive textbook. Philadelphia: Lippincott Williams \& Wilkins p. 2417-2429.

22. Yagi K (1996) Evolution of Lennox-Gastaut syndrome: a long-term longitudinal study. Epilepsia 37 Suppl 3: 48-51.
23. Ogawa K, Kanemoto K, Ishii Y, Koyama M, Shirasaka Y, et al. (2001) Longterm follow-up study of Lennox-Gastaut syndrome in patients with severe motor and intellectual disabilities: with special reference to the problem of dysphagia. Seizure 10: 197-202.

24. Hoffmann-Riem M, Diener W, Benninger C, Rating D, Unnebrink K, et al. (2000) Nonconvulsive status epilepticus--a possible cause of mental retardation in patients with Lennox-Gastaut syndrome. Neuropediatrics 31: 169-174.

25. Kohsaka S, Mizukami S, Kohsaka M, Shiraishi H, Kobayashi K (2002) Widespread activation of the brainstem preceding the recruiting rhythm in human epilepsies. Neuroscience 115: 697-706.
This article was originally published in a special issue, Epilepsy: Current Trends handled by Editor(s). Dr. Espinosa PS, Centro Internacional en Neurociencias, USA 